\title{
Large Eddy Simulations of the Entropic Lattice Boltzmann Method
}

\author{
Qiang Liu ${ }^{1, a^{*}}$, Yan Wang ${ }^{2, b}$ and Wei Xie ${ }^{1, c}$ \\ ${ }^{1}$ China Ship Development and Design Center, Wuhan 430064, China \\ ${ }^{2}$ Wuhan Ship Development Design Institute, Wuhan 430064, China \\ aliuqiang7012012@126.com, 'b310010100@hrbeu.edu.cn, xiewei@public.wh.hb.cn
}

Keywords: Entropic lattice Boltzmann method, turbulence, large eddy simulation, robustness Abstract. The large eddy simulation (LES) has not been adequately developed in the entropic lattice Boltzmann method (ELBM) framework. In particular, analysis of high Reynolds number turbulence simulations using ELBM-LES remains to be done. A standard Smagorinsky subgrid scale model in large eddy simulation based on the entropic lattice Boltzmann method was established. Simulations of high Reynolds number turbulence were carried out. The validity and robustness of ELBM-LES were explored for high Reynolds number turbulence simulations. Conclusion: ELBM-LES presented a good effectiveness and robustness in simulations of strong nonlinear turbulent flows at the high Reynolds number range.

\section{Introduction}

Lattice Boltzmann method (LBM) can be considered as a special discrete format of a simplified form of the Boltzmann equation in the kinetic theory of gases [1]. Since the Boltzmann equation was built on probability calculations of a molecular state and statistical theories, it was decided the lattice Boltzmann method is essentially a mesoscopic method. LBM describes materials from the level of distribution function which is between the micelles level and the molecular level. Characteristics of the clear physical background and simple boundary treatments make LBM a very promising numerical scheme [2-4].

Despite the exponentially growing literature devoted to the LBM, its progress in studying turbulent flow is not fully satisfactory compared with its achievements in other aspects at present [5]. Direct simulations of turbulence using LBM without turbulence models are still limited. The most remarkable turbulence model in LBM is the large eddy simulation (LES). Eggels and Somers et al. [6, 7] associated the turbulent stress tensor with the equilibrium distribution function in LBM, and carried out a result of large eddy simulation in earlier time. Comparison of the experimental data and simulation results including the mean flow field and the turbulence intensity indicated a satisfactory agreement. Studies of other LES subgrid scale models also demonstrated the potentiality of LBM in turbulence simulations. The dynamic subgrid scale model [8] and the inertial range consistent subgrid scale model [5] are among these concerned LES subgrid scale models. Lacking studies at present are twofold: the high Reynolds number turbulence simulation and more corresponding application researches. It appears that large eddy simulation has not been adequately developed in the LBM framework.

The shortcoming of the LB approach is the numerical instabilities that arise when the flow velocity or its spatial gradient becomes too large for a given lattice, resulting in negative values of the distribution function [9]. One of significant improvements is the entropic lattice Boltzmann method (ELBM) which led to stable explicit algorithms. However, more studies and analysis of the integrality of ELBM and LES remain to be done. We tried to use ELBM and LES to simulate high Reynolds number turbulence at $\mathrm{Re}=10^{6} \sim 10^{7}$ in this paper. In addition, the validity, applicability and robustness of ELBM-LES in turbulence simulations were also explored. 


\section{Theory of the entropic lattice Boltzmann method}

LBM equations reflect the collision-evolution law of particles, and similarly equations of ELBM can be written as Eq. 1:

$$
\begin{gathered}
f_{i}\left(\boldsymbol{r}+\boldsymbol{e}_{\alpha} \delta_{t}, t+\delta_{t}\right)-f_{i}(\boldsymbol{r}, t)=\alpha \beta\left[f_{i}^{\mathrm{eq}}(\boldsymbol{r}, t)-f_{i}(\boldsymbol{r}, t)\right] \\
H\left(f+\alpha\left(f^{\mathrm{eq}}-f\right)\right)=H(f) \\
v=c_{\mathrm{s}}^{2}\left(\frac{1}{2 \beta}-\frac{1}{2}\right)
\end{gathered}
$$

Where the distribution function $f$ is a function of space vector $\boldsymbol{r}(x, y, z)$, molecular velocity vectors $\boldsymbol{\xi}\left(\boldsymbol{\xi}_{x}, \boldsymbol{\xi}_{y}, \boldsymbol{\xi}_{z}\right)$ and time $t, \boldsymbol{e}_{\alpha}$ represents the discrete velocity model, $\delta_{t}$ represents the space of time, $f_{\alpha}^{\text {eq }}$ is the equilibrium distribution function, $v$ is the kinematic viscosity, $c_{\mathrm{s}}$ represents the lattice sound velocity.

The discrete form of $H$ function is written as Eq. 2:

$$
H\left(f_{i}\right)=\sum_{i=1}^{Q} f_{i} \ln \left(\frac{f_{i}}{\omega_{i}}\right)
$$

Where $\omega_{i}$ represents the corresponding weight factor.

The equilibrium distribution function used is shown in Eq. 3:

$$
f_{i}^{\mathrm{eq}}=\rho W_{\alpha} \prod_{\alpha=1}^{3}\left(2-\sqrt{1+3 u_{i}^{2}}\right)\left(\frac{2 u_{i}+\sqrt{1+3 u_{i}^{2}}}{1-u_{i}}\right)^{e_{\alpha, i}}
$$

Where $W_{\alpha}$ is the weight factor.

\section{Simulation of the flow around a 3-D inclined ellipsoid}

High Reynolds number turbulence simulation study of ELBM-LES is carried out for the flow around an inclined ellipsoid at $\mathrm{Re}=4.0 \times 10^{6}$. As shown in Fig. 1, the 3-D ellipsoid with an axial length ratio of 3:1 is inclined 10 degrees. The D3Q15 discrete velocity model and a nonequilibrium extrapolation scheme of boundary conditions were used. The mesh size is $1 / 30$ of the minor axis length $b$.

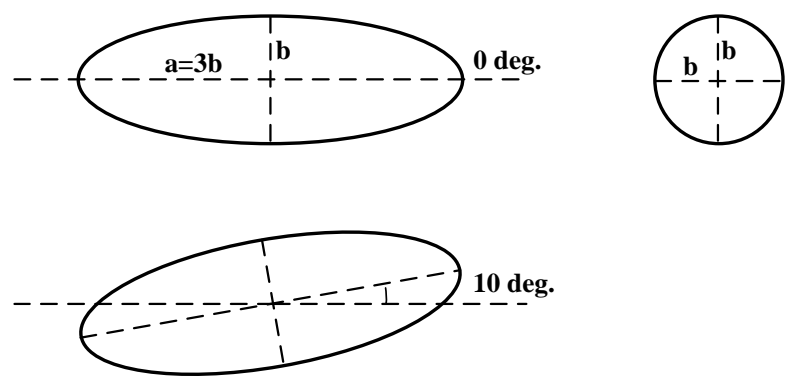

Fig. 1 The inclined ellipsoid

Pressure coefficients along the uppermost side and the lowermost side of ellipsoid surface including the experimental data of Clarke et al. [10] are respectively shown in Fig. 2 and Fig. 3 in which $L$ stands for the major axis length of ellipsoid and $X_{\mathrm{bc}}$ stands for the distance from center to surface point. 


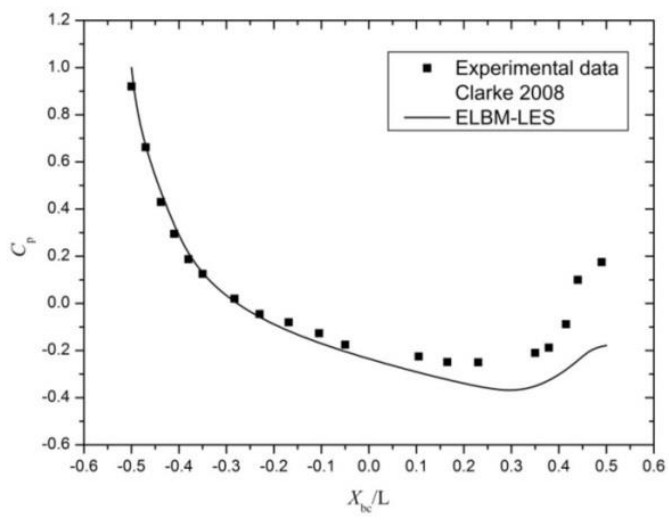

Fig. 2 Pressure coefficient distribution along the uppermost side of the ellipsoid surface

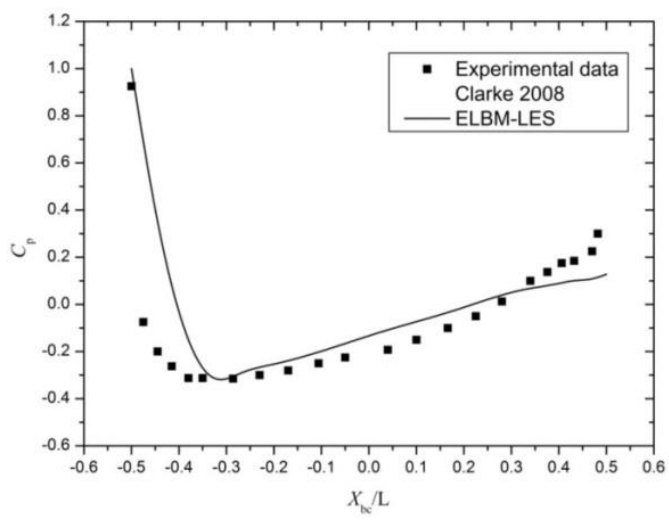

Fig. 3 Pressure coefficient distribution along the lowermost side of the ellipsoid surface

Simulated velocity magnitude and vorticity isosurface in the flow field are shown in Fig. 4 and Fig. 5 .

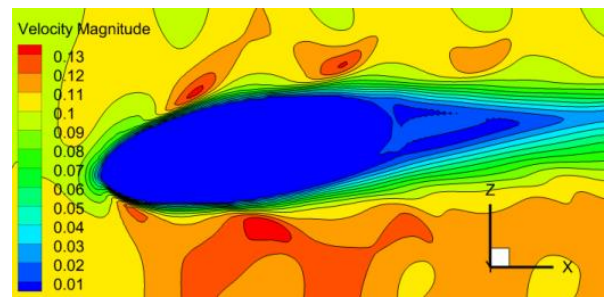

Fig. 4 Velocity magnitude in the longitudinal section
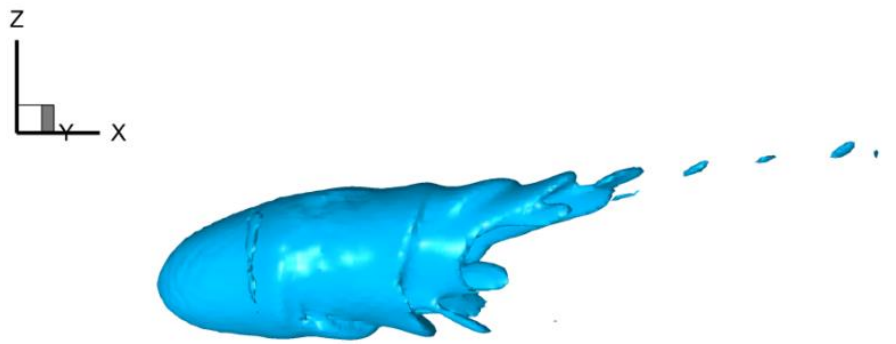

Fig. 5 Vorticity isosurface of the value of 0.01 in flow around the inclined ellipsoid 


\section{The robustness study of ELBM-LES}

In order to explore the applicability and robustness of ELBM-LES in high Reynolds number turbulence simulations, more complicated turbulent flows were simulated including the flow around two spheres and flow around a sphere with a vertical square plate. The simulated Reynolds number are both $\mathrm{Re}=1.0 \times 10^{7}$. The D3Q15 discrete velocity model and a nonequilibrium extrapolation scheme of boundary conditions were used. The mesh size is $1 / 30$ of sphere diameter.

Results are shown in Fig. 6-Fig. 9.

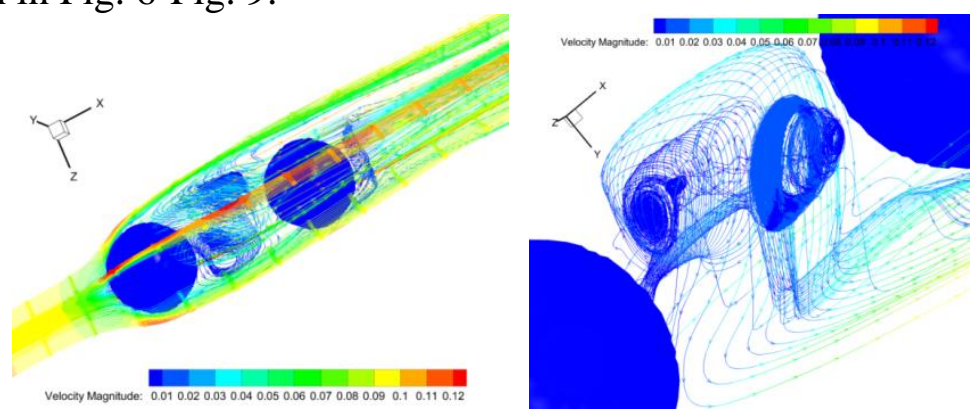

Fig. 6 Streamline distribution of the flow around two spheres

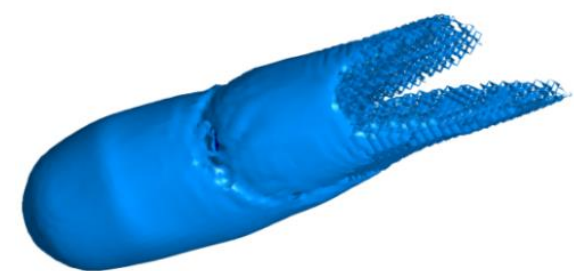

Fig. 7 Vorticity isosurface of the value of 0.013 in flow around two spheres
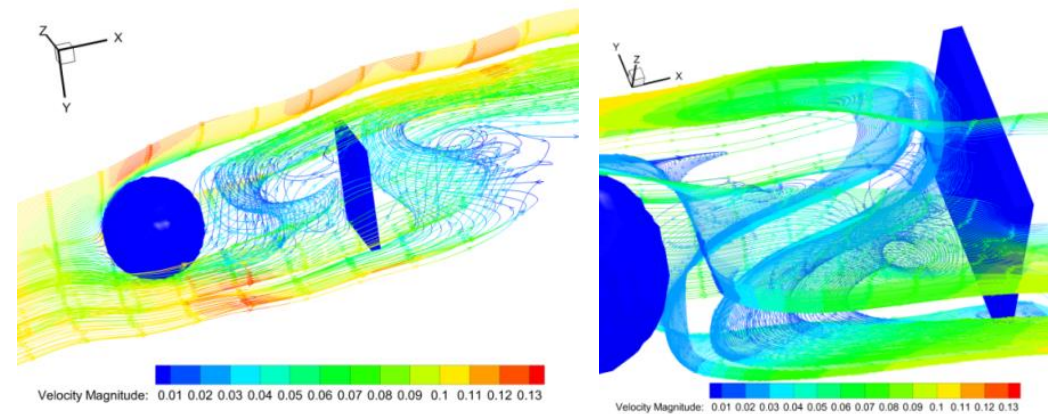

Fig. 8 Streamline distribution of the flow around a sphere with a vertical square plate

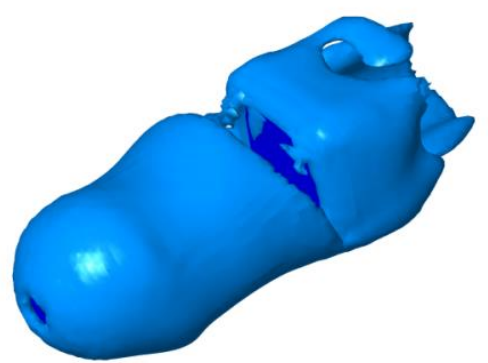

Fig. 9 Vorticity isosurface of the value of 0.013 in flow around a sphere with a vertical square plate 


\section{Conclusions}

Turbulence is a strong nonlinear flow phenomenon. The purpose of this study was to explore the effectiveness and robustness of ELBM-LES in high Reynolds number turbulence simulations. Turbulent flow fields simulated include the flow around a 3-D inclined ellipsoid, flow around two spheres and flow around a sphere with a vertical square plate.

(1) Practices of numerical simulations above prove that the entropic lattice Boltzmann method is a highly stable numerical scheme in simulating nonlinear turbulence. After the introduction of subgrid scale model in LES, ELBM become an effective high Reynolds number turbulence simulation tool.

(2) Simulations of the flow around two spheres and flow around a sphere with a vertical square plate reflect a satisfactory robustness of ELBM-LES. Such robustness led us to believe that introductions of other LES models to the entropic lattice Boltzmann method are considered also have good prospects.

(3) ELBM-LES can effectively simulate the high Reynolds number turbulence and more potential applications could be achieved based on the combination of ELBM and LES.

\section{References}

[1] X. He, L.S. Luo, Theory of the lattice Boltzmann method: From the Boltzmann equation to the lattice Boltzmann equation, Physical Review E 56 (1997) 6811-6817.

[2] Y. Huo, Z. Rao, The numerical investigation of nanofluid based cylinder battery thermal management using lattice Boltzmann method, International Journal of Heat and Mass Transfer 91 (2015) 374-384.

[3] S. Bogner, R. Ammer, U. Rüde, Boundary conditions for free interfaces with the lattice Boltzmann method, Journal of Computational Physics 297 (2015) 1-12.

[4] M. Kaneda, T. Haruna, K. Suga, Ghost-fluid-based boundary treatment in lattice Boltzmann method and its extension to advancing boundary, Applied Thermal Engineering 72 (2014) 126-134.

[5] Y.H. Dong, P. Sagaut, S. Marie, Inertial consistent subgrid model for large-eddy simulation based on the lattice Boltzmann method, Physics of Fluids 20 (2008) 035104.

[6] J.G.M. Eggels, J.A. Somers, Numerical simulation of free convective flow using the lattice-Boltzmann scheme, J. Heat Fluid Flow 16 (1995) 357-364.

[7] J.G.M. Eggels, Direct and large-eddy simulation of turbulent fluid flow using the lattice Boltzmann scheme, Int. J. Heat Fluid Flow 17 (1996) 307-323.

[8] K.N. Premnath, M.J. Pattison, S. Banerjee, Dynamic subgrid scale modeling of turbulent flows using lattice-Boltzmann method. Physica A 388 (2009) 2640-2658.

[9] C.K. Aidun, J.R. Clausen, Lattice-Boltzmann method for complex flows, Annu. Rev. Fluid Mech. 42 (2010) 439-472.

[10]D.B. Clarke, P.A. Brandner, G.J. Walker, Experimental and computational investigation of flow around a 3-1 prolate spheroid, WSEAS Transactions on Fluid Mechanics 3 (2008) 207-217. 